\title{
Cobalt-doped bioceramic scaffolds fabricated by 3D printing show enhanced osteogenic and angiogenic properties for bone repair
}

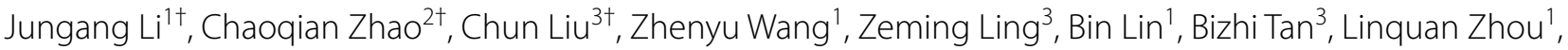

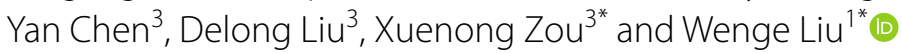

\section{${ }^{*}$ Correspondence:}

zxnong@hotmail.com;

sxsuperman@163.com

†Jungang Li, Chaogian

Zhao, and Chun Liu have

contributed equally to this

work

${ }^{1}$ Department

of Orthopaedics, Fujian

Medical University Union

Hospital, Fuzhou 350001

China

${ }^{3}$ Guangdong Provincial Key Laboratory of Orthopaedics and Traumatology,

Department of Spine Surgery, The First Affiliated Hospital of Sun Yat-Sen University, Guangzhou 510080, China Full list of author information is available at the end of the article

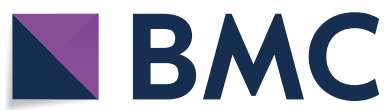

(c) The Author(s), 2021. Open Access This article is licensed under a Creative Commons Attribution 4.0 International License, which permits use, sharing, adaptation, distribution and reproduction in any medium or format, as long as you give appropriate credit to the original author(s) and the source, provide a link to the Creative Commons licence, and indicate if changes were made. The images or other third party material in this article are included in the article's Creative Commons licence, unless indicated otherwise in a credit line to the material. If material is not included in the article's Creative Commons licence and your intended use is not permitted by statutory regulation or exceeds the permitted use, you will need to obtain permission directly from the copyright holder. To view a copy of this licence, visit http:// creativecommons.org/licenses/by/4.0/. The Creative Commons Public Domain Dedication waiver (http://creativecommons.org/publi cdomain/zero/1.0/) applies to the data made available in this article, unless otherwise stated in a credit line to the data. 
Keywords: Cobalt, Bioceramics, Angiogenesis, Osteogenesis, Bone defect repair

\section{Background}

Currently, the reconstruction of bone defects associated with tumors, trauma, inflammation, and surgery represents a challenge for clinicians in orthopedic practice. To date, autologous bone grafts are still considered the optimal standard for the regeneration of bone defects [1]. However, disadvantages, such as inadequate bone sources and donor site morbidity, have limited its applications in practice. Allogeneic and xenogeneic bone grafts, as an alternative to autografts, can avoid these shortcomings mentioned above. However, the high risks of immunological rejection and pathogen transmission impose a major restriction on their clinical application $[2,3]$.

Artificial bone grafts have been developed to solve the above problems due to their advantages of readily availability, superior stability, and desired reproducibility [4]. Standing out among artificial bone grafts, bioceramics, particularly beta-tricalcium phosphate $(\beta-\mathrm{TCP})$, exhibit desired biocompatibility, good degradability, and remarkable osteoconductivity and osteoinductivity [5]. $\beta$-TCP has recently drawn substantial attention and further become a hopeful artificial bone graft alternative $[5,6]$. In vitro studies showed that bone marrow mesenchymal stem cells (BMSCs) seeded on $\beta$-TCP were stimulated to proliferate and differentiate into osteoblasts $[7,8]$. In vivo trials, with bone remodeling, $\beta$-TCP was gradually degraded and eventually displaced by mature new bone $[5,9]$. Nevertheless, disadvantages such as poor angiogenic properties severely limit its applications.

Angiogenesis, generally regarded as a challenge for artificial bone grafts, is vital for osteogenesis, and artificial bone grafts should be modified to enable angiogenesis and thus improve osteogenesis [10]. Blood vessels not only recruit osteoprogenitors to the graft surface, but also furnish them with both nutrients and minerals for cell survival and mineralization [11, 12]. Understanding the relationship between angiogenesis and osteogenesis would be of great significance to improve bone regeneration.

Cobalt (Co), known for simulating hypoxic environment, was found to stabilize hypoxia-inducible factor 1- $\alpha$ (HIF-1a) from degradation by inactivating proline hydroxylase (PHD) under normal oxygen pressure. The hypoxic environment can activate HIF-1a pathway cascades and then upregulate the expression of targeted genes such as vascular endothelial growth factor (VEGF) [13]. VEGF is not only beneficial to angiogenesis, but also essential for bone development and regeneration [14]. Modification by doping Co into biomaterials, such as calcium phosphate coatings, hydroxyapatite, and bioactive glass, had positive effects on angiogenesis and osteogenesis in vitro and enhanced bone regeneration in vivo [15-18].

We previously fabricated crystalline $\mathrm{Ca}_{10} \mathrm{Li}\left(\mathrm{PO}_{4}\right)_{7}$ (CLP) by doping lithium (Li) into $\beta$-TCP substrate, and the results revealed that CLP possessed relatively higher compressive strength and better biocompatibility than pure $\beta$-TCP [19]. To improve the angiogenic properties of CLP, we hypothesized that CLP doped with $\mathrm{Co}^{2+}$ ions could achieve excellent osteogenic and angiogenic properties while maintaining a certain compressive strength to promote bone regeneration. In addition to the composition of artificial bone grafts, the structure fabricated by different techniques will also affect its performance. Slurry extrusion 3D printing (SE-3DP) has been applied 
to manufacture porous scaffolds with precise, dimensionally controlled and orderly spatial microstructures, including porosity properties [20, 21].

In this study, we investigated the osteogenic and angiogenic effects of CLP doped with $\mathrm{Co}^{2+}$ ions. Porous Co-doped CLP scaffolds were prepared by the solid-phase sintering method and SE-3DP techniques [19]. The effects of different Co concentrations in CLP on its physicochemical properties, biocompatibility, and osteogenic and angiogenic properties were systematically investigated in vitro. At 8 weeks after scaffold implantation, microcomputed tomography (micro-CT) and histological methods were utilized to analyze the in vivo bone regeneration of Co-doped scaffolds (as displayed in Fig. 1). We believe that our findings will have great significance for clinical application in the bone repair field.

\section{Results}

\section{Characterization of the Co $(0,1,2.5,5,10)$ scaffolds}

In the present study, Co-doped scaffolds were synthesized by doping CLP with various concentrations of Co $(0,0.1,0.25,0.5,1 \mathrm{~mol} \%)$ and SE-3D printing techniques, named Co0, Co1, Co2.5, Co5, and Co10 scaffolds. The effect of Co doping on the CLP structure was determined by characterizing the Co $(0,1,2.5,5,10)$ scaffolds via X-ray diffraction (XRD), Raman spectrophotometry, and scanning electron microscope (SEM). Figure $2 \mathrm{~A}$ shows the XRD patterns of the Co $(0,1,2.5,5,10)$ scaffolds. The XRD patterns of the Co $(1,2.5,5,10)$ scaffolds were found to be similar to that of $\mathrm{Co} 0$ and were devoid of any additional peaks corresponding to Co. With increasing Codoping content, the XRD profile moved toward a slight right shift as a whole. When the doping percentage of $\mathrm{Co}^{2+}$ ions increased up to $1 \mathrm{~mol} \%$, the crystal planes (110), (128), $(4,0,10)$, and $(2,0,20)$ obviously changed.

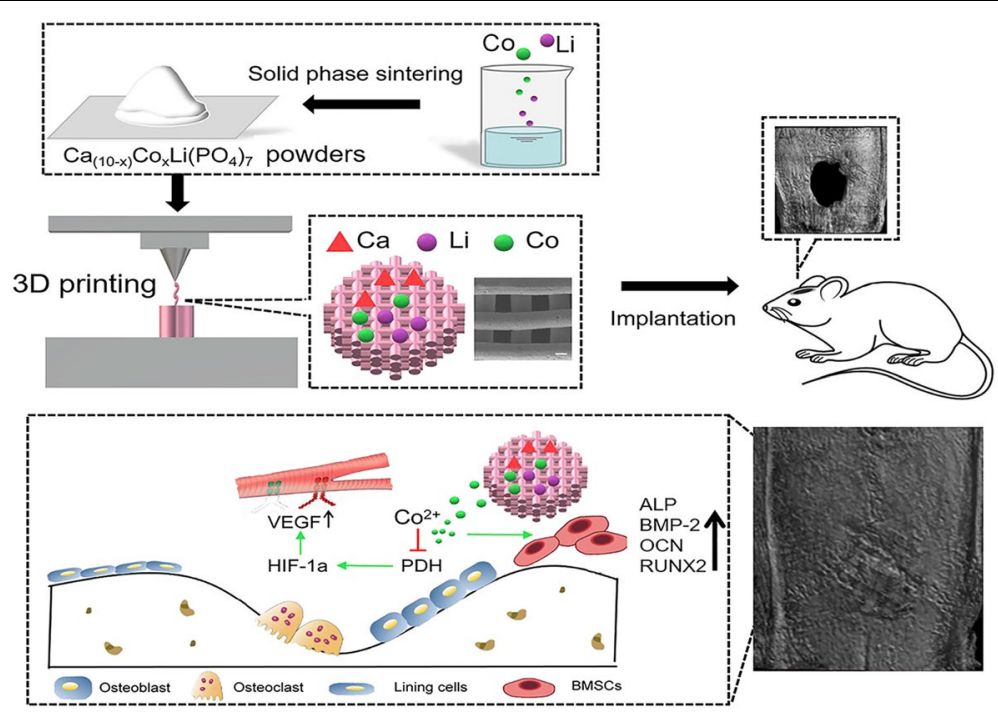

Fig. 1 Schematic illustration of the fabrication of porous bioceramic scaffolds doped with different amounts of cobalt to promote bone defect repair via enhanced osteogenic and angiogenic properties 

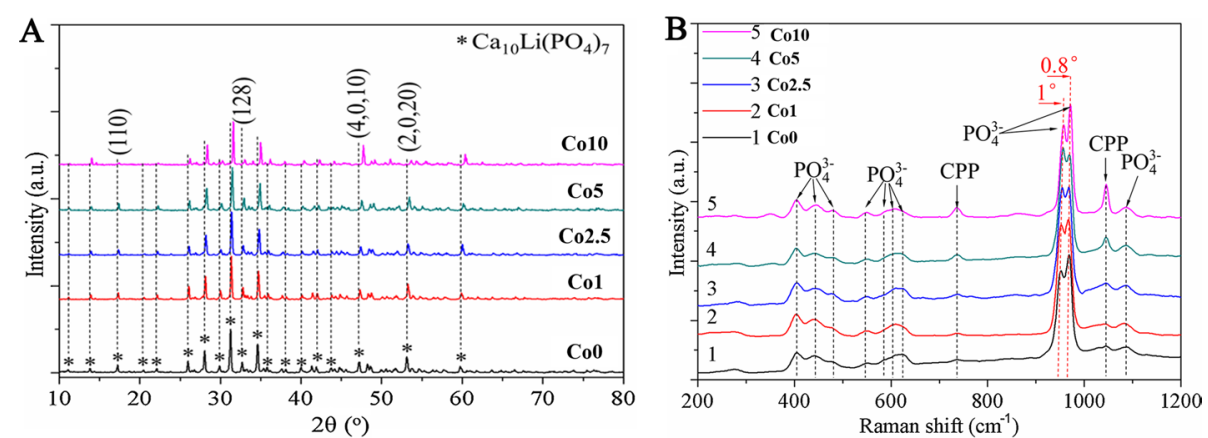

Fig. 2 Characterization of the Co $(0,1,2.5,5,10)$ scaffolds. A XRD patterns and $\mathbf{B}$ Raman spectrum of the Co $(0,1,2.5,5,10)$ scaffolds

Figure 2B displays the Raman spectra of the Co $(1,2.5,5,10)$ scaffolds. Compared with the Co0 scaffold, the band positions of the Co $(1,2.5,5,10)$ scaffolds were altered, moving to the direction of a higher Raman shift with increasing Co-doping level.

In Fig. 3A, a, color difference was observed between the Co-doped and -undoped scaffolds. After adding $\mathrm{Co}^{2+}$ ions to CLP, the color of the scaffolds transformed from white to purple. With increasing Co content, the color of the scaffolds successively changed from white $(\mathrm{Co} 0)$ to light purple (Co1) to purple (Co2.5) to dark purple (Co5) and to dark reddish purple (Co10). Figure $3 \mathrm{~A}$ and B demonstrates the SEM surface morphology of the Co $(0,1,2.5,5,10)$ scaffolds. Based on the micrographs, regular pores and cylindrical wires of scaffolds fabricated by SE-3DP were interlaced in each layer. The printing wire and pore size were approximately $500 \mu \mathrm{m}$ and $400 \mu \mathrm{m} \times 400 \mu \mathrm{m}$, respectively. At high magnification (Fig. 3B), the crystal size of the Co $(0,1,2.5,5)$ scaffolds decreased gradually with increasing Co dopant. When the doping percentage was up to $1 \mathrm{~mol} \%$, some melted crystals and microcracks were observed on the Co10 scaffold surface. In Fig. 3C, energy-dispersive spectroscopy (EDS) results showed peaks of Co within the Co $(1,2.5,5,10)$ scaffolds. However, there was no characteristic peak of Co in the Co0 scaffold. In addition, the measured $\mathrm{Co} /(\mathrm{Ca}+\mathrm{Co})$ ratios enhanced with increasing Co-doping amount. The EDS results also revealed that $\mathrm{Co}^{2+}$ ions were successfully incorporated into Co $(1,2.5,5,10)$ scaffolds.

\section{In vitro degradation and mineralization properties of the $\operatorname{Co}(0,1,2.5,5,10)$ scaffolds}

To study the effect of Co doping on the physicochemical properties of CLP, degradation and mineralization tests were performed. Figure $4 \mathrm{~A}$ and $\mathrm{B}$ shows the degradation properties of the $\mathrm{Co}(0,1,2.5,5,10)$ scaffolds in Tris- $\mathrm{HCl}$ solution. As shown in Fig. 4A, the weight loss of the Co $(0,1,2.5,5,10)$ scaffolds tended to increase with the extension of soaking time. At days 21 and 28, the weight loss of the Co $(0,1,2.5,5,10)$ scaffolds also raised with increasing Co-doping concentration. Meanwhile, the weight loss of the Co $(1,2.5,5,10)$ scaffolds was higher than that of Co0 scaffolds, and the differences were significant $(p<0.01)$. Figure $4 \mathrm{~B}$ shows that the $\mathrm{pH}$ value of Tri-HCL solutions in the Co $(0,1,2.5,5,10)$ groups revealed a synchronously ascending state over the prolonged soaking time.

Figure 4C-E shows the surface morphology of the Co $(0,1,2.5,5,10)$ scaffolds after mineralization in vitro. As displayed in Fig. $4 \mathrm{D}$ and $\mathrm{E}$, the Co $(0,1,2.5,5)$ scaffolds 


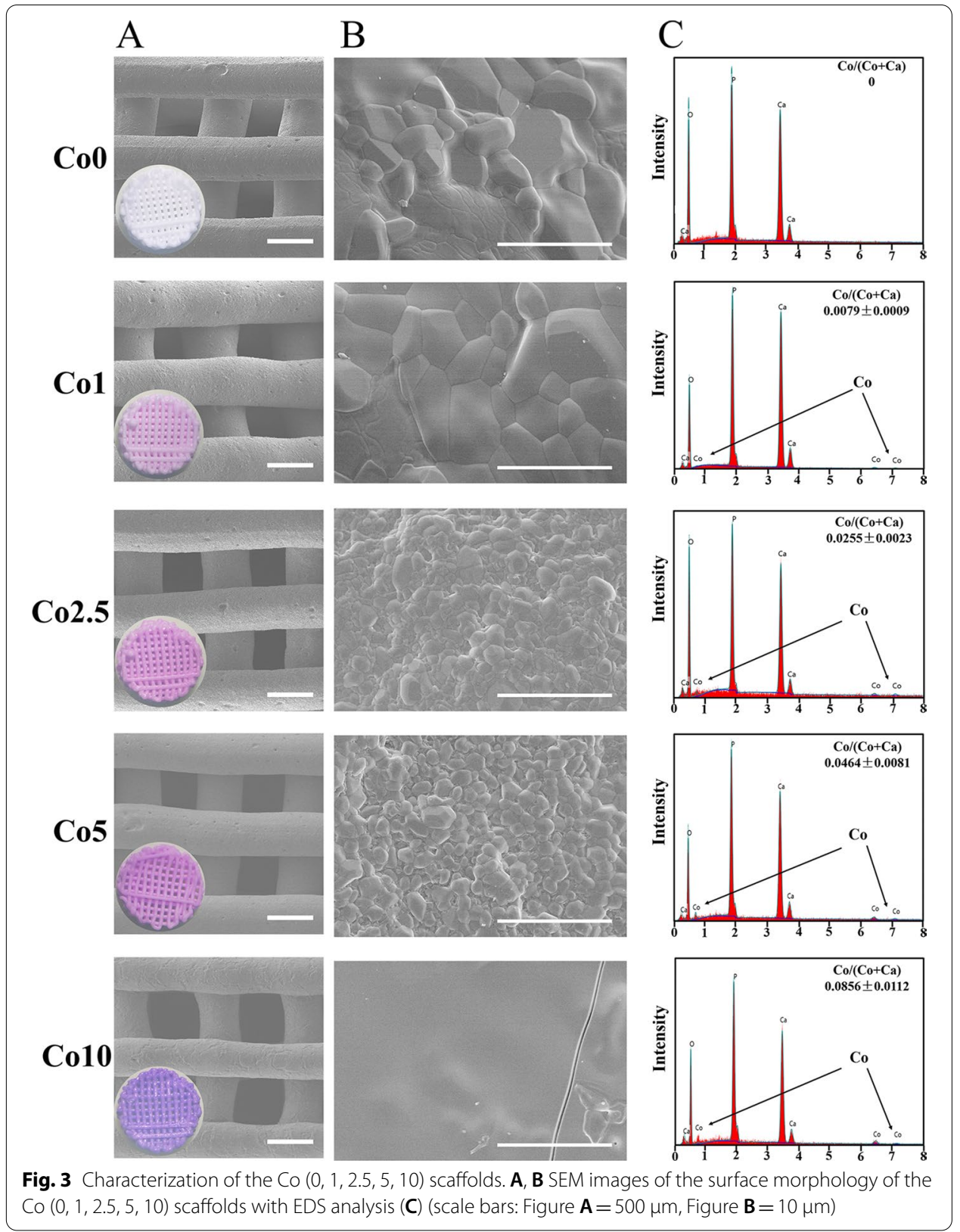

showed apparent mineralization effects in vitro. A particulate and loose microstructure was consistently dispersed on the surface of the Co $(0,1,2.5,5)$ scaffolds, and cracks were observed. The XRD data (Fig. 4F) demonstrated that the mineralization phases of the Co $(0,1,2.5,5)$ scaffolds are hydroxyapatite. However, the Co10 scaffolds showed none of the changes mentioned above.

\section{Mechanical properties of the Co $(0,1,2.5,5,10)$ scaffolds}

The mechanical properties of the Co $(0,1,2.5,5,10)$ scaffolds were obtained by compressive strength testing. As shown in Fig. 4G, the compressive strength of the Co $(1,2.5$, 5) scaffolds slightly decreased with increasing $\mathrm{Co}^{2+}$ ion content compared with the $\mathrm{CoO}$ 


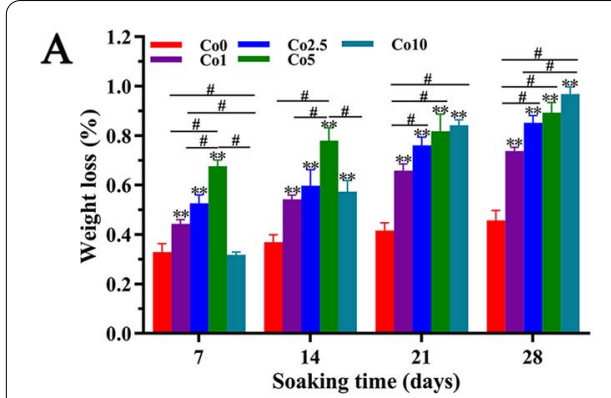

B
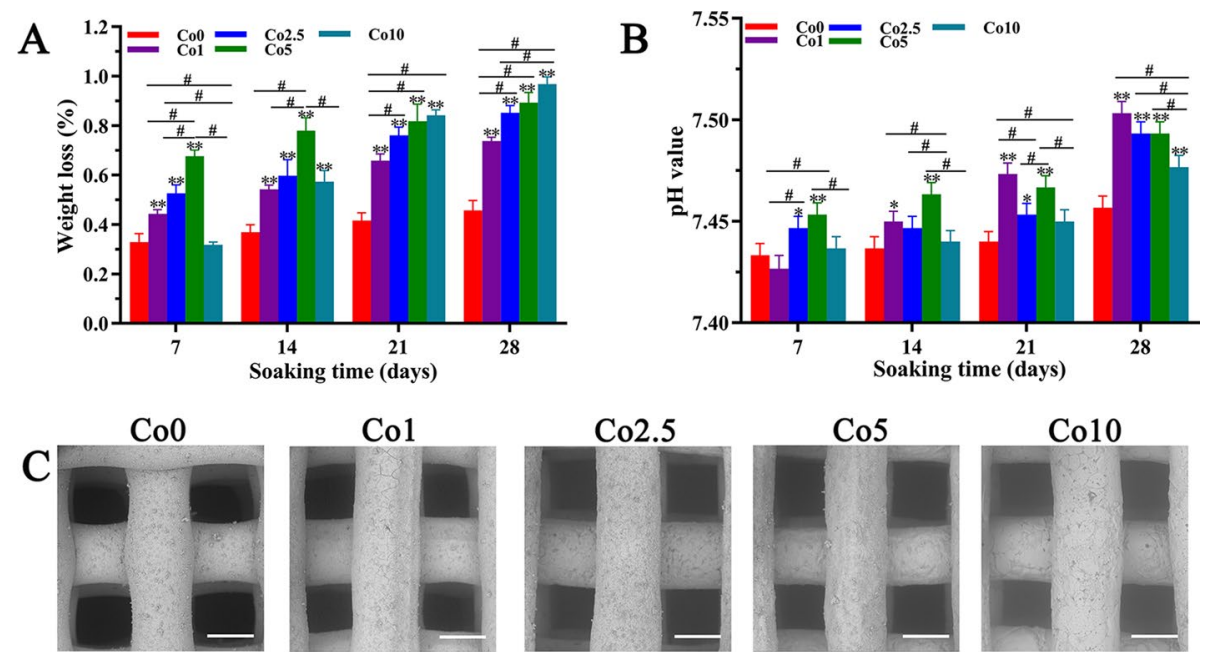

Co1
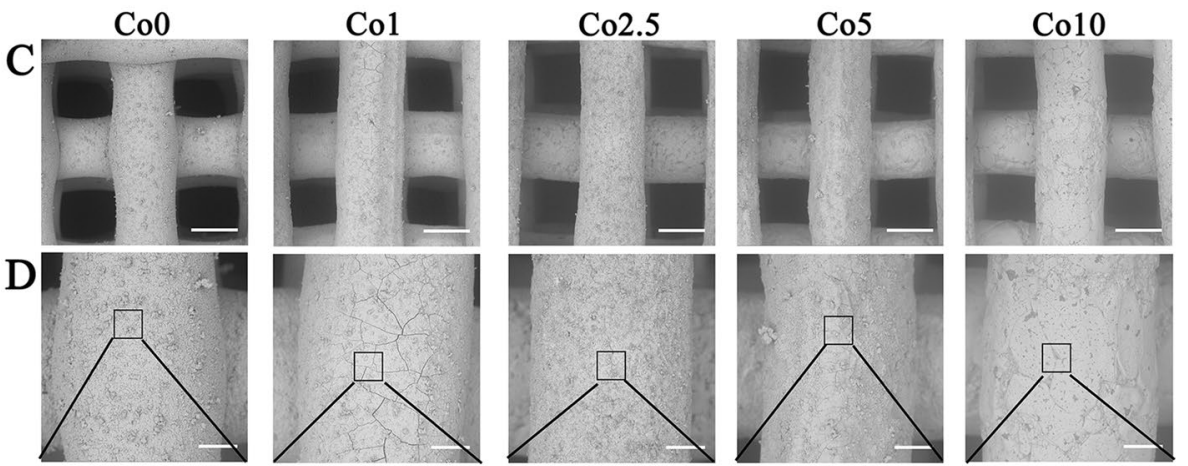

$\mathrm{E}$
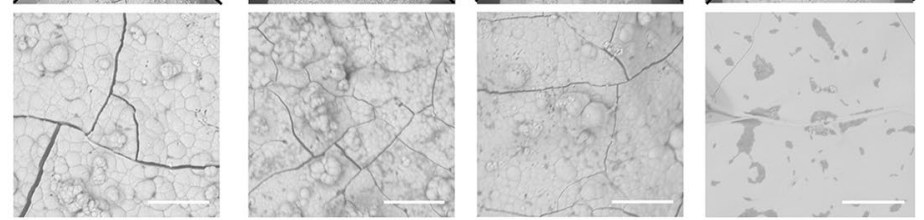

$\mathrm{F}$

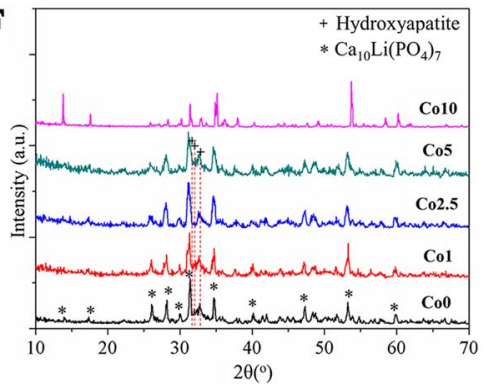

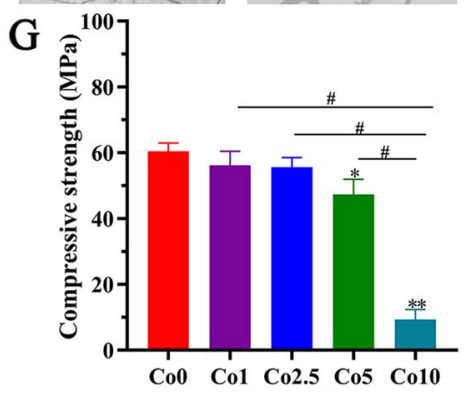

Fig. 4 In vitro physicochemical properties of the $\operatorname{Co}(0,1,2.5,5,10)$ scaffolds. A Weight loss of the Co $(0,1$, $2.5,5,10)$ scaffolds. $\mathbf{B}$ pH value of Tris- $\mathrm{HCl}$ solution after soaking the $\mathrm{Co}(0,1,2.5,5,10)$ scaffolds. $\mathbf{C}$-E SEM images of the surface microstructure of the $\operatorname{Co}(0,1,2.5,5,10)$ scaffolds after mineralization in vitro (scale bars: Figure $\mathbf{C}=300 \mu \mathrm{m}$, Figure $\mathbf{D}=100 \mu \mathrm{m}$, Figure $\mathbf{E}=20 \mu \mathrm{m}) . \mathbf{F}$ XRD patterns of the Co $(0,1,2.5,5,10)$ scaffolds after mineralization in vitro. $\mathbf{G}$ Compressive strength of the $\mathrm{Co}(0,1,2.5,5,10)$ scaffolds. The data are expressed as the mean \pm SD. ${ }^{*} p<0.05$ and ${ }^{* *} p<0.01$, comparison with the Co0 group; ${ }^{*} p<0.05$, comparison among the Co $(1,2.5,5,10)$ groups

scaffolds; remarkably, the compressive strength of the Co10 scaffold sharply declined, and the differences between the Co10 group and the other groups were significant $(p<0.01)$.

\section{Biocompatibility of the Co $(0,1,2.5,5,10)$ scaffolds}

Preliminary evaluation of the biocompatibility on the Co $(0,1,2.5,5,10)$ scaffolds was detected by means of a cytotoxicity assay for subsequent biological studies. As shown in 
Fig. $5 \mathrm{~A}$, the cell viability in the Co $(0,1,2.5,5)$ groups was $103.31 \%, 103.03 \%, 106.17 \%$, and $79.25 \%$, which were all greater than $70 \%$ and did not show obvious cytotoxicity, while the cell viability in the Co10 group was $69.94 \%$, which was less than $70 \%$ and displayed obvious cytotoxicity.

To further assess the biocompatibility of the Co $(0,1,2.5,5)$ scaffolds, the proliferation of rat BMSCs (rBMSCs) on the Co $(0,1,2.5,5)$ scaffolds was measured. As shown in Fig. 5B, the OD values in the Co $(0,1,2.5,5)$ groups increased with the extension of

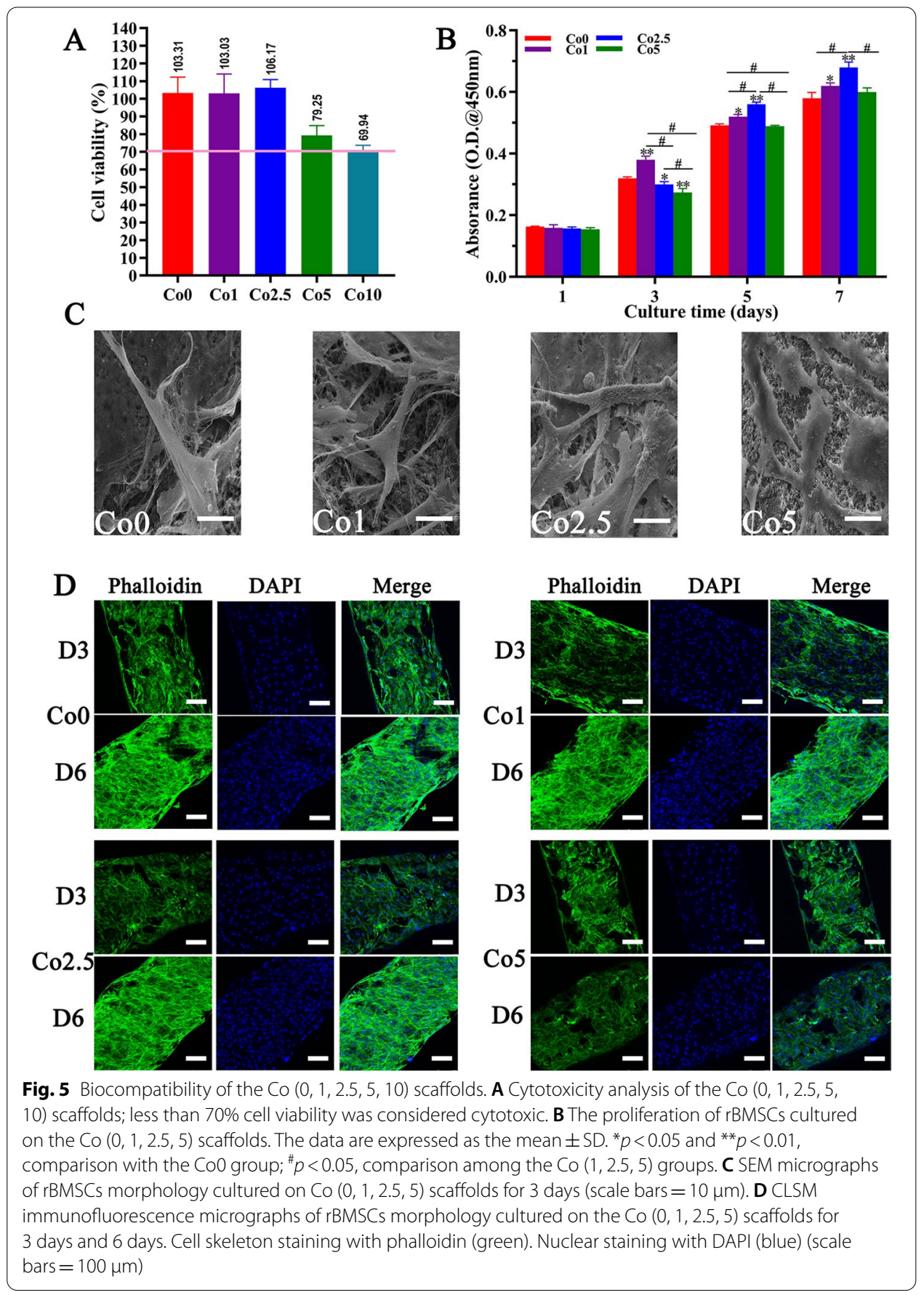


culture time, which indicated that rBMSCs cultured on the Co $(0,1,2.5,5)$ scaffolds proliferated well. In particular, after $24 \mathrm{~h}$ of incubation, there were no significant differences in cell proliferation among the Co $(0,1,2.5,5)$ scaffolds. At day 3 , the proliferation of rBMSCs on the Co1 scaffold showed a significant difference from that on the Co $(0,2.5$, 5) scaffolds, whereas cell proliferation on the Co $(2.5,5)$ scaffolds was less than that on $\mathrm{Co} 0$ scaffold. Interestingly, as the culture time increased, the Co2.5 scaffold effectively promoted rBMSCs proliferation at days 5 and 7 compared with the Co $(0,1,5)$ scaffolds. However, the Co5 scaffold showed a slight decrease in cell proliferation compared with the other Co-doped scaffolds at each time point.

The adhesion and morphology of rBMSCs on the Co $(0,1,2.5,5)$ scaffolds were also observed. Figure $5 \mathrm{C}$ and D shows SEM images of rBMSCs at day 3 and confocal laser scanning microscope (CLSM) images of rBMSCs at days 3 and 6 after seeding on the Co $(0,1,2.5,5)$ scaffolds, respectively. As revealed by the SEM images in Fig. 5C, the rBMSCs on Co the $(0,1,2.5)$ scaffolds attached and spread well with more abundant filopodia than those on the Co5 scaffold. No obvious distinction was noted in cell morphology on the Co $(0,1,2.5)$ scaffold surfaces. Intriguingly, the CLSM results presented a similar trend as the Cell Counting Kit-8 (CCK-8) assay data and SEM images. In the CLSM results, cell skeleton was stained green, and the nucleus was stained blue. As shown in Fig. 5D, there were few rBMSCs on the surface of the Co $(0,1,2.5,5)$ scaffolds at day 3 , and there were more rBMSCs at day 6 , as observed by CLSM images. However, compared with the Co $(0,1,2.5)$ scaffolds, the Co5 scaffold had slightly fewer cells at each time point.

\section{In vitro angiogenic properties of the $\mathrm{Co}(0,1,2.5,5)$ scaffolds}

The angiogenic properties of the Co $(0,1,2.5,5)$ scaffolds were investigated by conducting an in vitro tubule formation assay. As shown in Fig. 6A, human umbilical vein endothelial cells (HUVECs) in all groups sprouted and self-assembled to form branched nodes and circles after $4 \mathrm{~h}$ of culture, finally forming continuous tubular networks. It is worth noting that the Co2.5 group induced the most tube-like structures among all of the groups. After $6 \mathrm{~h}$ of culture, the Co2.5 group maintained its advantage in stimulating tubule formation. Although the HUVECs began to undergo apoptosis after $16 \mathrm{~h}$ of culture, which was the case in all of the groups, the cells in the Co2.5 group displayed a better network structure than the cells in Co the $(0,1,5)$ groups. Quantitative analysis of the branches number (Fig. 6B) and total branching length (Fig. 6C) showed significantly higher values in $\mathrm{Co}(1,2.5,5)$ groups than that in the $\mathrm{Co} 0$ groups, especially in the $\mathrm{Co} 2.5$ group.

HIF- $1 \alpha$ and VEGF expression in rBMSCs incubated with Co $(0,1,2.5,5)$ extracts was also analyzed. As exhibited in Fig. 6D and E, both HIF-1 $\alpha$ and VEGF expression was significantly upregulated in the Co $(1,2.5,5)$ groups compared with that of the Co0 group. In particular, the Co2.5 group showed greatly enhanced expression of HIF- $1 \alpha$ and VEGF in comparison with that of the Co 1 and Co 5 groups.

In vitro osteogenic properties of the $C_{0}(0,1,2.5,5)$ scaffolds

The osteogenic properties of the Co $(0,1,2.5,5)$ scaffolds were measured by alkaline phosphatase (ALP) staining and alizarin red staining. As shown in Fig. 7A, after culture 


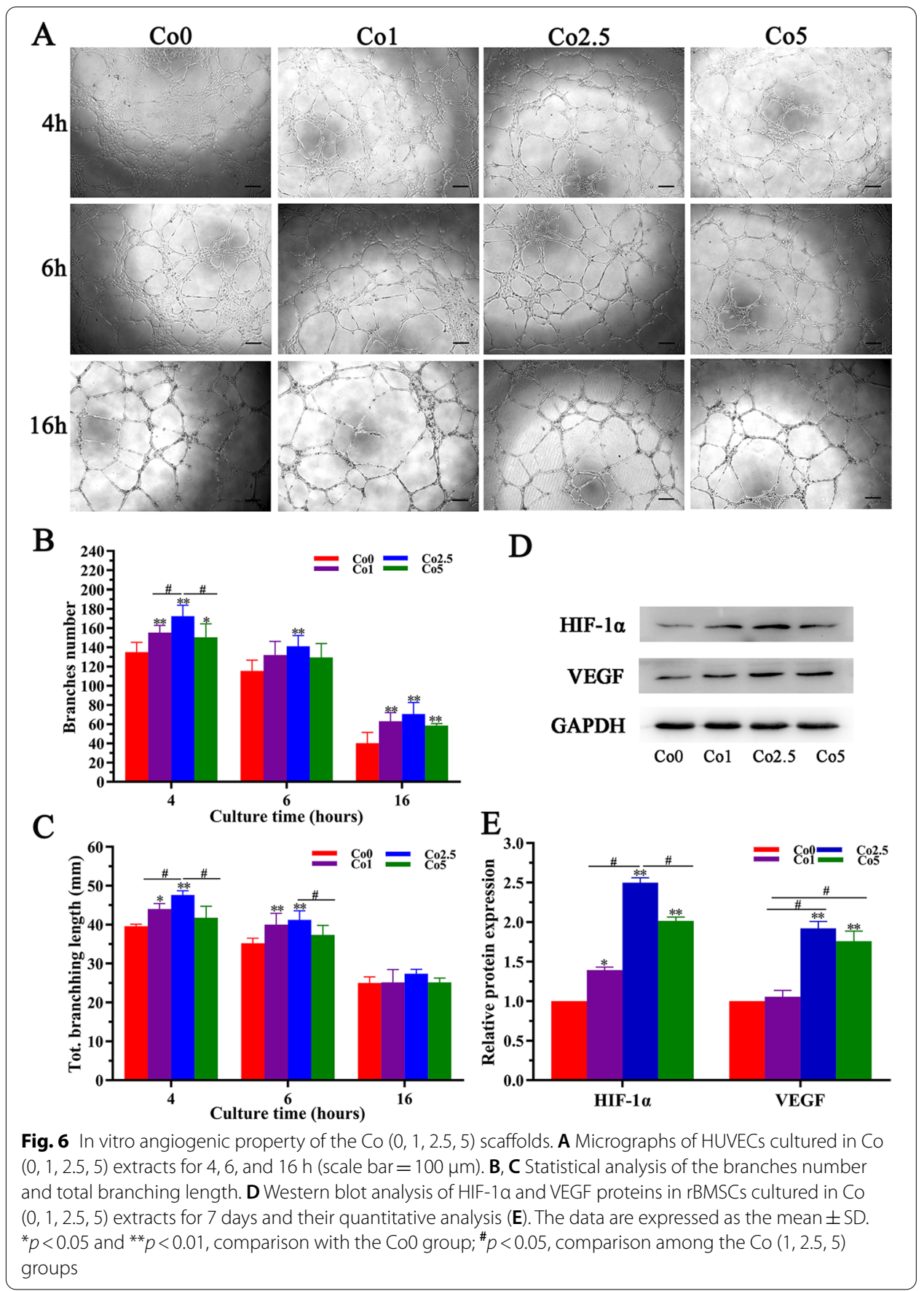

for 7 days, the intensity of ALP staining in the Co $(0,1,2.5,5)$ groups was similar to that in the osteogenic medium (OM) group. With prolonged culture time, the staining intensity in all groups on day 14 was higher than that on day 7. Moreover, the Co2.5 group showed more intense staining than the other groups. With the exception of the initial osteogenic marker, the terminal state of osteogenic differentiation of rBMSCs was examined via alizarin red staining. As displayed in Fig. 7B, alizarin red staining showed that a large number of red-stained calcium nodules were formed in all groups, and the calcium 


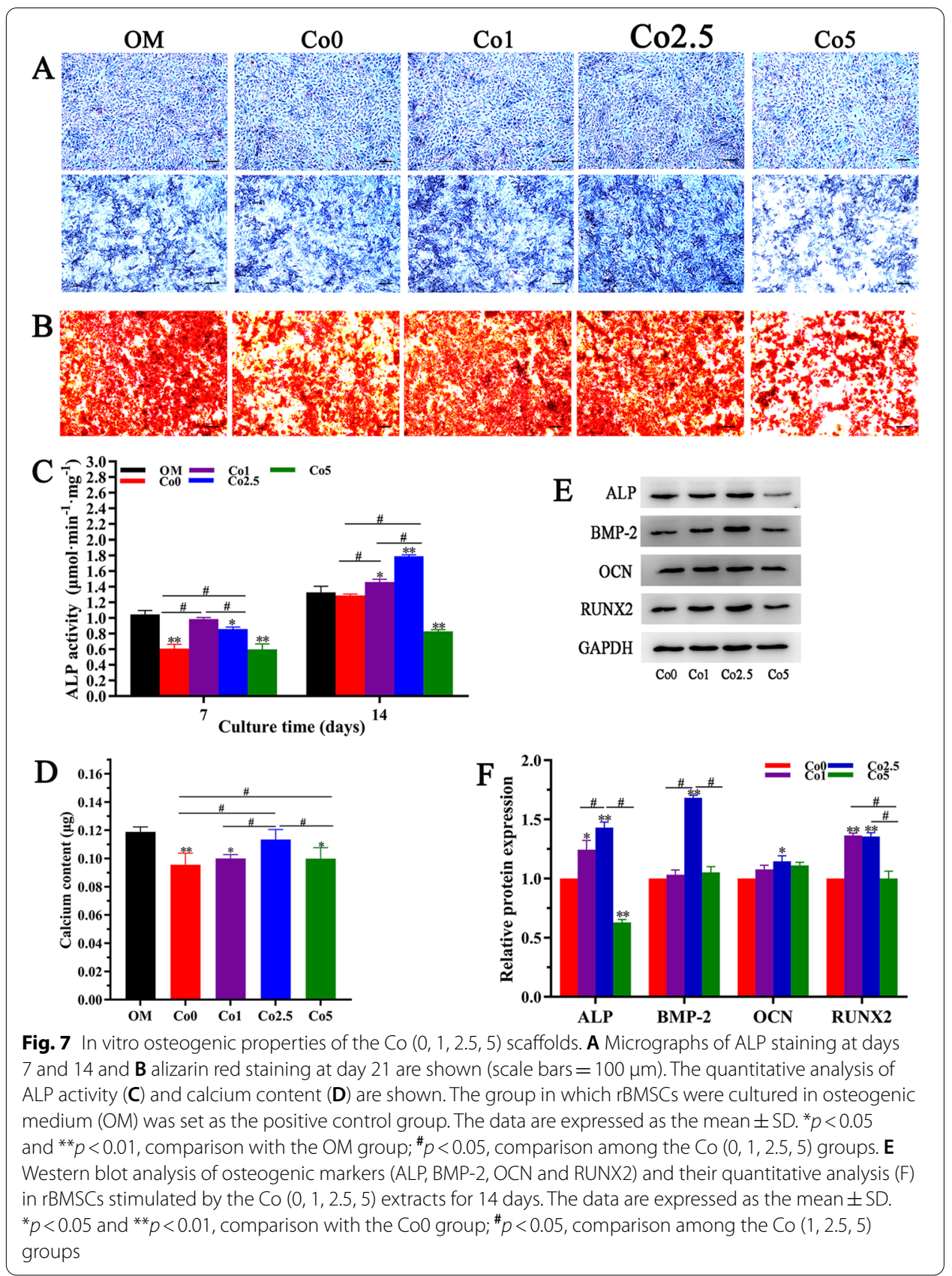

nodule numbers in the Co $(1,2.5)$ groups and OM group were significantly higher than those in the Co $(0,5)$ groups. To quantitatively evaluate the osteogenic properties of the Co $(0,1,2.5,5)$ scaffolds, the ALP activity and calcium content were analyzed. As revealed in Fig. $7 \mathrm{C}$, the ALP activity in the Co $(0,1,2.5,5)$ groups was lower than that in the OM group over 7 days of culture. Additionally, the Co1 group enhanced ALP activity among the $\mathrm{Co}(0,1,2.5,5)$ groups. Interestingly, the Co2.5 group exhibited markedly increased ALP activity compared to with the other groups over 14 days of stimulation, the difference was significant $(p<0.05)$. As shown in Fig. 7D, the group exposed to the Co2.5 extract had higher calcium contents than the groups exposed to the Co $(0,1,5)$ 
extracts. Although the OM group had a higher calcium content, the differences between the Co2.5 and OM groups were not significant $(p>0.05)$.

The expression levels of osteogenic markers, including ALP, bone morphogenetic protein-2 (BMP-2), runt-related transcription factor 2 (RUNX2), and osteocalcin (OCN), in rBMSCs cultured in Co $(0,1,2.5,5)$ extracts at day 14 were also examined. As shown in Fig. 7E and F, the Co2.5 group exhibited a more significant upregulation of the expression of ALP and BMP-2 than the Co $(0,1,5)$ groups at day 14 . Although OCN expression did not show a significant difference among the Co $(1,2.5,5)$ groups, OCN expression in the Co2.5 group was higher than that in the Co0 group. RUNX2 expression in the Co2.5 group at day 14 was also higher than that in the Co $(0,5)$ groups but slightly lower than that in the Co1 group, which showed no noticeable difference $(p>0.05)$.

\section{In vivo bone regeneration of the Co2.5 scaffold}

A cranium bone defect model with $5 \mathrm{~mm}$ in diameter in rats was constructed to validate the in vivo osteogenic properties of Co-doped scaffolds. Since the in vitro results revealed that the osteogenic and angiogenic properties of the Co2.5 scaffold were superior to those of the Co $(0,1,5)$ scaffolds, the Co2.5 scaffold was applied to investigate whether it resulted in enhanced bone regeneration in vivo.

At 8 weeks after implantation, CT-constructed images were obtained to evaluate the extent of new bone formation by micro-CT. As shown in Fig. 8A, the round bone defect in the blank group clearly existed, and a small amount of bone-like tissue could be observed on the edge of the defect. In the $\mathrm{Co} 0$ group, scaffolds in bone defects were visible in situ, and the edge of the scaffold was covered by bone-like tissue. Notably, the scaffold in the Co2.5 group was almost integrated with the surrounding normal bone tissue and was not easily visible, and the scaffold-host bone interface was completely fused. In addition, as displayed in planar views (Fig. 8B), a larger amount of new bone along the margin and in the center of the defect was formed in the Co2.5 group compared with the $\mathrm{Co} 0$ and blank groups. Quantitative evaluation of new bone formation was performed using a bone volume/tissue volume (BV/TV) assay. As shown in Fig. 8C, the BV/TV was the highest in the $\mathrm{Co} 2.5$ group, followed by the $\mathrm{Co} 0$ group and blank group. Compared

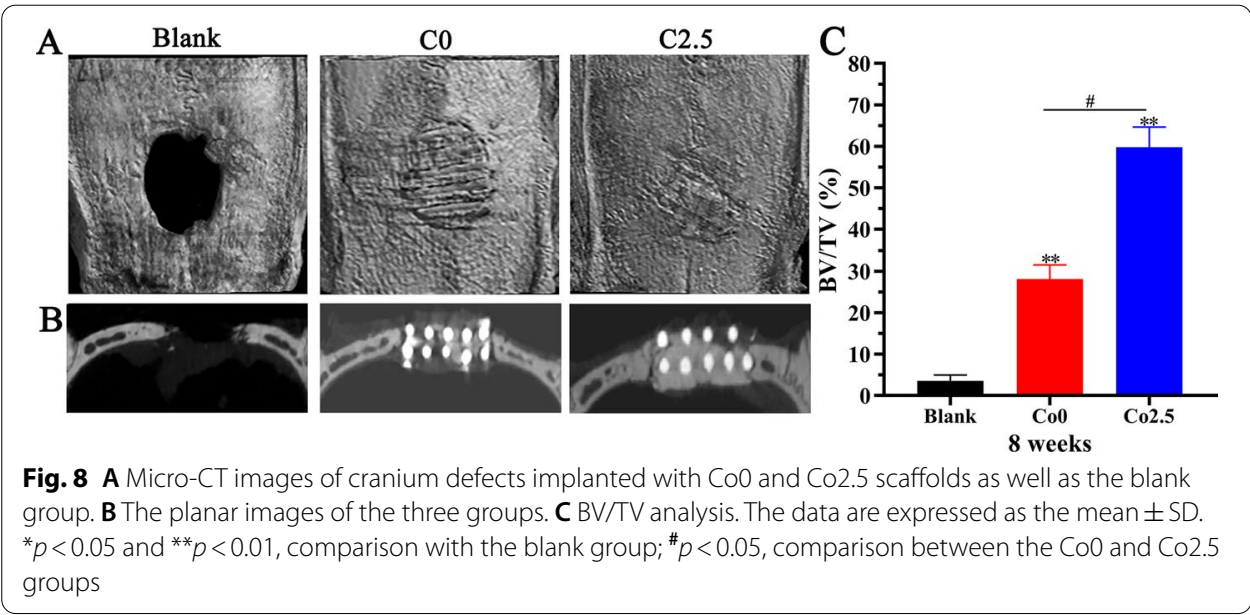


with that in the blank and $\mathrm{Co} 0$ groups, the $\mathrm{BV} / \mathrm{TV}$ in the Co2.5 group was significantly increased $(p<0.01)$.

Following micro-CT analysis, the samples were performed histologically to assess new bone formation. The hematoxylin and eosin (H\&E) staining data indicated a bone regeneration tendency parallel to the above micro-CT data. New bone tissue was stained with different shades of red in H\&E staining. As revealed in Fig. 9A and B, new bone tissue was found in the center and along with the margin of the defect in the Co2.5 group, but was relatively rare in the $\mathrm{Co} 0$ group, with the least new bone formation in the blank group. Furthermore, the new bone area was evaluated quantitatively. As displayed in Fig. 9C, the new bone area was significantly increased in Co2.5 group, followed by the $\mathrm{Co} 0$ and blank groups, and the differences among groups were significant $(p<0.01)$. Additionally, Masson's trichrome staining was implemented to histologically evaluate the maturity of new bone tissue. Masson's trichrome staining showed that collagen fibers and immature bone tissue were blue, and mature bone tissue was red. As shown by Masson's trichrome staining in Fig. 9D and E, in the blank group, only a small amount of collagen fibers and immature bone tissue were formed at the defect edge. Although the new bone tissue formed in the $\mathrm{Co} 2.5$ group and $\mathrm{Co} 0$ group was mainly mature bone tissue, the amount of new bone tissue in the Co2.5 group was significantly greater than that in the $\mathrm{Co} 0$ group.

\section{Discussion}

The doping of inorganic ions into bioceramics is an effective strategy for modification, and has attracted extensive attention as a burgeoning avenue for promoting bone regeneration $[22,23]$. In the present study, Co-doped scaffolds were synthesized by the solid-phase sintering method and SE-3DP. A color difference was observed between the Co-doped and -undoped scaffolds. The color of the $\mathrm{Co} 0$ scaffold was white, whereas the Co $(1,2.5,5,10)$ scaffolds turned purple with increasing Co-doping amount. Color

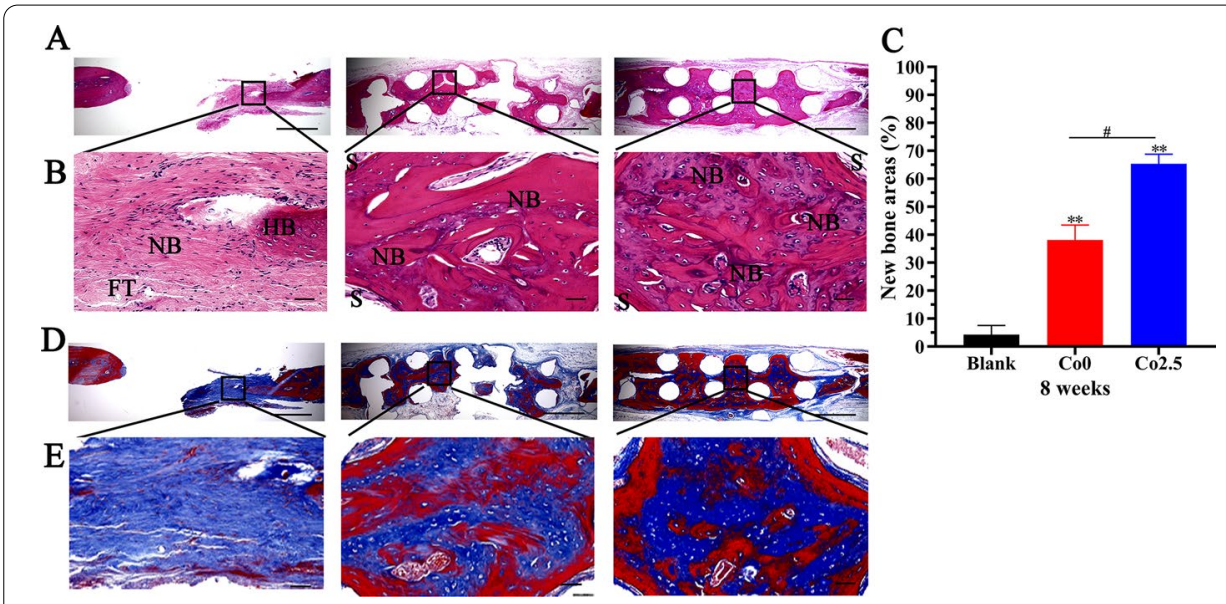

Fig. 9 A, B H\&E staining images of three groups (scale bar: Figure $A=50 \mu \mathrm{m}$, Figure $B=100 \mu \mathrm{m}$, NB: new bone, HB: host bone, FT: fibrous tissue, S: scaffolds). C New bone areas were calculated by Image-Pro Plus 6.0 software. The data are expressed as the mean \pm SD. ${ }^{*} p<0.05$ and ${ }^{* *} p<0.01$, comparison with the blank group; ${ }^{p} p<0.05$, comparison between the $\mathrm{Co} 0$ and Co2.5 groups. D, E Masson's trichrome staining images (scale bar: Figure $\mathbf{D}=50 \mu \mathrm{m}$, Figure $\mathbf{E}=100 \mu \mathrm{m}$ ) 
variation provided a clue that $\mathrm{Co}^{2+}$ ions were successfully doped into CLP. Doping was investigated with the purpose of replacing $\mathrm{Ca}^{2+}$ ions from the CLP with $\mathrm{Co}^{2+}$ ions. Ion doping can cause variations in the microstructure and crystal morphology of bioceramics depending on the property, size, and amount of the dopant [24]. The XRD patterns of the Co $(1,2.5,5,10)$ scaffolds were almost identical to that of the Co0 scaffold, and there was no additional peak corresponding to $\mathrm{Co}$, which indicated that $\mathrm{Co}^{2+}$ ions might have been completely doped into the CLP structure. With increasing Co-doping content, the movement of the XRD profile toward a slight right shift might be attributed to the substitution of the low ionic radius $\mathrm{Co}^{2+}(0.070 \mathrm{~nm})$ for the high ionic radius of $\mathrm{Ca}^{2+}(0.099 \mathrm{~nm})$, which may cause the contraction of the crystal lattice $[16,25,26]$. The ionic radius mismatch of the doped ions mentioned above may also explain why the crystal planes (110), (128), $(4,0,10)$, and $(2,0,20)$ changed when the doping percentage of $\mathrm{Co}^{2+}$ ions increased up to $1 \mathrm{~mol} \%$. The variation in crystal planes is usually closely related to a reduction in crystallinity and crystal size. Hence, it can be concluded that partial replacement of $\mathrm{Ca}^{2+}$ ions with $\mathrm{Co}^{2+}$ ions may lead to smaller crystal sizes and reduced crystallinity, which can account for the changes in crystal size on Co the $(0,1$, $2.5,5,10)$ scaffolds' surface. Similarly, the Raman spectra of the Co $(1,2.5,5,10)$ scaffolds also showed that Co doping may result in a decrease in the lattice constant of CLP [27]. Together, Co doping has a certain influence on CLP structure, and the greater the doping amount is, the greater the influence, especially as the Co-doping concentration increased to $1 \mathrm{~mol} \%$.

The degradation and resorption of artificial bone grafts play a significant role in the bone repair process [28], which occurs in parallel with osteogenesis and bone regeneration. As artificial bone grafts are degraded, new bone tissue grows into bone defect sites to complete bone repair. The data in Fig. 4A demonstrate that Co-doped CLP possessed excellent degradation properties compared with Co undoped CLP, which was beneficial for accelerating bone formation. Additionally, the mineralization potential of artificial bone grafts contributes to the enhanced bone-bonding process. The mineralization results showed that the Co $(1,2.5,5)$ scaffolds had mineralization effects similar to those of the $\mathrm{Co} 0$ scaffold, which was conducive to firm bone binding to host bone after implantation in vivo. However, the Co10 scaffold showed no mineralization properties, which may be attributed to distortion of the crystal lattice caused by partial replacement of $\mathrm{Ca}^{2+}$ ions with $\mathrm{Co}^{2+}$ ions.

A higher compressive strength presents a higher fracture toughness [29]. Although the compressive strength of the Co $(1,2.5,5,10)$ scaffolds decreased with increasing $\mathrm{Co}^{2+}$ ion content in comparison with the Co0 scaffold, especially the Co10 scaffold, these data with respect to the Co $(1,2.5,5)$ scaffolds remained in the range of human trabecular bone [30], suggesting that the Co $(1,2.5,5)$ scaffolds could also bear external stress and resist fracture. The possible reason accounting for the low mechanical properties of the Co10 scaffold is that doping with $\mathrm{Co}^{2+}$ ions lead to increased lattice distortion of CLP, resulting in large penetrating cracks. Additionally, a desired artificial bone graft should an exhibit an interconnected porous structure, in which the pore diameters should be between 200 and $500 \mu \mathrm{m}$, and the porosity should be above 50\% [31, 32]. An ideal interconnected porous structure not only maintains certain mechanical properties, but also favors cell penetration, angiogenesis, and osteogenesis [31, 32]. In this work, the 
interconnected pore size was approximately $400 \mu \mathrm{m} \times 400 \mu \mathrm{m}$, and the porosity was $40 \%$ (Fig. 3A), slightly less than the designated porosity due to printing precision issues and the sintering process. However, the ideal scaffold must compromise and balance porosity and compressive strength. In general, the doping of $\mathrm{Co}^{2+}$ ions have a certain influence on the compressive strength, degradation, and mineralization properties of CLP, and the greater the doping amount is, the greater the influence.

As degradation proceeds, the Co-doped scaffolds could release $\mathrm{Co}^{2+}$ ions into the surrounding environment; the amount released mainly depends on the concentration of $\mathrm{Co}$ incorporated into the scaffolds. Related reports have confirmed that $\mathrm{Co}^{2+}$ ions at high concentrations may cause cell and tissue damage [33]. With this in mind, we preliminarily detected the cytotoxicity of the Co $(0,1,2.5,5,10)$ scaffolds for subsequent biological studies. As shown in Fig. 5A, the Co $(0,1,2.5,5)$ scaffolds did not show obvious cytotoxicity (cell viability $>70 \%$ ), while the Co10 scaffold displayed obvious cytotoxicity (cell viability $<70 \%$ ). The reason for cytotoxicity on the Co10 scaffold may be that doping Co has a significant influence on the degradation performance of CLP, and the greater the doping amount, the more obvious the degradation, especially the Co10 scaffold. As the degradation of the Co10 scaffold increases, the amount of released ions increases accordingly. According to the results of the characterization, physicochemical properties, compressive strength, and cytotoxicity assay, we selected the Co $(0,1,2.5,5)$ scaffolds for subsequent in vitro research.

Although the Co $(0,1,2.5,5)$ scaffolds showed no significant cytotoxicity, it was necessary to further assess the biocompatibility of the Co $(0,1,2.5,5)$ scaffolds. It is vital that osteoprogenitors proliferate well on artificial bone grafts in bone regeneration and bone remodeling processes [34]. Therefore, we cultured rBMSCs on Co $(0,1,2.5,5)$ scaffolds and measured cell proliferation. The CCK- 8 results demonstrated that rBMSCs could proliferate well on the Co $(0,1,2.5,5)$ scaffolds over the culture time, particularly on the Co2.5 scaffold, showing favorable biocompatibility. The prerequisite that artificial bone grafts exert biological functions is that rBMSCs can easily adhere and spread on the surface of artificial bone grafts. As far as the artificial bone graft is concerned, its components and surface morphology will have a certain impact on cell adhesion. SEM images revealed that rBMSCs were healthy spread and attached well on the surface of the Co $(0$, $1,2.5)$ scaffolds with abundant filopodia compared with the Co5 scaffold. Intriguingly, the CLSM results presented a similar trend as the CCK- 8 assay data and SEM images, i.e., rBMSCs could attach and proliferate well on the Co $(0,1,2.5,5)$ scaffolds over the culture time. However, the Co5 scaffold showed a slight decrease in cell proliferation compared with the other Co-doped scaffolds at each time point, which may be attributed to the fact that although the cell viability of the Co5 scaffold was slightly greater than the critical value of $70 \%$ (cell viability $79 \%>70 \%$ ) and showed no obvious cytotoxicity, it may still had a certain influence on the metabolic state of rBMSCs. In summary, all of these results clearly confirmed that, among the Co $(0,1,2.5,5)$ scaffolds, the Co2.5 scaffold possesses excellent biocompatibility, which is one of the requirements of artificial bone grafts.

The principal reason for incorporating $\mathrm{Co}^{2+}$ ions in artificial bone grafts is dependent on the inherent property of $\mathrm{Co}^{2+}$ ions to accelerate angiogenesis [35]. $\mathrm{Co}^{2+}$ ions, a chemical inducer, can deactivate PHD, improving the HIF-1 $\alpha$ concentration and therefore 
imitating hypoxic conditions under normal oxygen tension [36]. In turn, HIF-1 $\alpha$ can upregulate the expression of angiogenic genes such as VEGF, which have close relationships with bone tissue development and regeneration [37]. In addition, VEGF, as a key regulator of the growth of the vascular network, can affect endothelial cells by stimulating their migration, proliferation, and eventually the formation of tubular structures [38]. Nevertheless, studies remain rare regarding whether incorporating Co into CLP can also induce a hypoxic response to promote angiogenesis, and thus enhance bone regeneration. Our results showed that HUVECs in the Co $(1,2.5,5)$ groups displayed a better network structure than those in the $\mathrm{Co} 0$ group, demonstrating that $\mathrm{Co}^{2+}$ ions released from Co $(1,2.5,5)$ scaffolds are beneficial to the formation of tube-like structures in HUVECs, especially in the Co2.5 group. Moreover, the Co2.5 group showed greatly enhanced expression of HIF- $1 \alpha$ and VEGF compared with that of the Co1 and Co5 groups, while the $\mathrm{Co} 0$ group showed no obvious effect on the expression of HIF-1 $\alpha$ and VEGF. These results indicated that the Co-doped CLP can simulate a hypoxic environment under normal oxygen pressure and thus promote VEGF expression to accelerate angiogenesis. Since the Co $(0,1,2.5,5)$ scaffolds have similar substrates, it is suggested that the release of $\mathrm{Co}^{2+}$ ions from the $\mathrm{Co}(1,2.5,5)$ scaffolds mainly contributes to the promotion of HIF- $1 \alpha$ and VEGF protein expression. Therefore, the incorporation of $\mathrm{Co}^{2+}$ ions into CLP is an available way to induce the hypoxic cascade to enhance angiogenesis for bone regeneration. Together with tubule formation data, the Co2.5 extract may be more conducive to angiogenesis than the Co $(0,1,5)$ extracts.

An ideal artificial bone graft should possess excellent osteogenic properties to accelerate bone repair. Acting as an extracellular matrix, the components of artificial bone grafts, including inorganics and organics, play a vital role in promoting osteogenic differentiation of osteoprogenitors. Hence, we qualitatively and quantitatively assessed the influence of the Co $(0,1,2.5,5)$ scaffolds on the osteogenic differentiation of rBMSCs in terms of initial and terminal osteogenic markers. The results indicated that the Co $(0,1$, $2.5,5)$ scaffolds could promote osteogenic differentiation of rBMSCs, and the osteogenic performance of Co2.5 scaffold was superior to that of the Co $(0,1,5)$ scaffolds.

The transitional stages of osteoprogenitors into osteoblasts involve the spatiotemporal expression of osteogenic genes, e.g., Runx 2 and OCN [39]. Therefore, the effect of the Co $(0,1,2.5,5)$ scaffolds on the expression levels of osteogenic markers, including ALP, BMP-2, RUNX2, and OCN, in rBMSCs was analyzed. These data further indicated that the Co2.5 scaffold could promote osteogenic markers to higher expression levels than the Co $(0,1,5)$ scaffolds. Some possible explanations for these findings are as follows. First, the stabilization of HIF-1a activated by $\mathrm{Co}^{2+}$ ions can induce the expression of a number of genes that are activated in fracture repair [40]. Second, $\mathrm{Co}^{2+}$ ions could also act as an agonist of $\mathrm{Ca}^{2+}$ ions and induce Wnt5 signal amplification, improving the expression of osteogenesis-related genes [41]. However, the expression of the aforementioned osteogenic genes did not increase with increasing Co-doping percentage. The reason might be the possibility of an antagonistic effect of $\mathrm{Co}^{2+}$ ions on $\mathrm{Ca}^{2+}$ ions at a high Co concentration and a reduction in expression [41].

Bone defect repair by artificial bone grafts involves a series of complex processes, and therefore, it is necessary to verify the bone regeneration of artificial bone grafts in vivo. Since the in vitro experiments demonstrated that the Co2.5 scaffolds showed excellent 
osteogenic and angiogenic properties compared with other scaffolds, the Co2.5 scaffold was employed to observe whether it also resulted in enhanced in vivo bone regeneration. The in vivo results demonstrated no significant new bone tissue formation in the blank group, indicating that the critical-sized defect model was suitable for evaluating the osteogenic capacity of scaffolds in vivo. Furthermore, the Co2.5 scaffold could significantly promote bone regeneration and had a positive effect on bone defect repair in comparison with the $\mathrm{Co} 0$ scaffold. It has been widely accepted that the local hypoxic environment is indispensable for bone regeneration. Since the Co2.5 and Co0 scaffolds are identical in the substrates and fabrication techniques, the sole discrepancy between the two scaffolds was the incorporation of $\mathrm{Co}^{2+}$ ions. $\mathrm{Co}^{2+}$ ions released from the $\mathrm{Co} 2.5$ scaffold can mimic hypoxic conditions by stabilizing HIF- $1 \alpha$ activity. Hypoxia plays an essential role in improving angiogenesis and osteogenesis by inducing the HIF-1 $\alpha$ pathway and promoting the migration, proliferation, differentiation, and VEGF secretion of MSCs [42, 43], thus promoting bone regeneration. On the other hand, the enhanced expression of VEGF, as one of the downstream regulators of HIF- $1 \alpha$, facilitates the angiogenic process and neovascularization of scaffolds. Taken together, the above findings suggested that adding $\mathrm{Co}^{2+}$ ions into CLP could improve bone regeneration by enhancing angiogenic and osteogenic capacities and is beneficial to bone defect repair.

However, there is another aspect to consider: most of the scaffolds appeared to remain after in vivo implantation for 8 weeks; in other words, the scaffolds showed limited signs of degradation despite undergoing a certain level of degradation. This finding was probably due to the short degradation time. Neo-bone formation could only occur through the surface and pores of scaffold within a limited time, while scaffold mostly remained without being replaced by neo-bone tissues. Although the Co2.5 scaffold partially degraded, the bone regeneration quality was excellent, demonstrating excellent bone defect repair properties. With respect to degradation, in-depth research may be required to optimize the scaffolds in the future. In short, this study showed that incorporating Co into CLP is an effective avenue to enhance the angiogenic and osteogenic properties of CLP. The Co2.5 scaffold is promising for use as an artificial bone graft with potentially improved bone regeneration.

\section{Conclusion}

In this study, porous scaffolds were fabricated by doping CLP with various concentrations of $\mathrm{Co}(0,0.1,0.25,0.5,1 \mathrm{~mol} \%)$ and SE-3D printing techniques, named $\mathrm{Co}$, Co1, Co2.5, Co5, and Co10. The incorporation of Co impacted the microstructure and physicochemical properties of CLP. The effect was enhanced along with increasing concentrations of Co doping and was especially evident in the Co10 scaffold. The in vitro data demonstrated that the Co $(1,2.5,5)$ scaffolds showed excellent biocompatibility and osteogenic and angiogenic properties compared with the CoO scaffold, especially the Co2.5 scaffold. In comparison with the Co0 scaffold, the Co2.5 scaffold significantly enhanced bone regeneration in rat calvarium defects at 8 weeks post-implantation. Together, our results suggest that the incorporation of Co into CLP is an available path to promote potential osteogenic and angiogenic properties. The Co2.5 scaffold holds promise for use as an artificial bone graft in bone repair with enhanced osteogenic and angiogenic potential. 


\section{Materials and methods} Materials

Polyvinyl alcohol, $\left(\mathrm{NH}_{4}\right)_{2} \mathrm{HPO}_{4}(99 \%), \mathrm{Ca}\left(\mathrm{NO}_{3}\right)_{2} \bullet 4 \mathrm{H}_{2} \mathrm{O}$ (99\%), Li3PO4 (99\%), $\mathrm{CoCl}_{2}$ (99\%), NH3.H2O (25-28\%), and Tris- $\mathrm{HCl}$ were purchased from Sinopharm Chemical Reagent Co., Ltd. Simulated body fluid (SBF) was purchased from Leagene (China). Minimum Eagle's medium (MEM), Dulbecco's modified Eagle's medium/Ham's F-12 (DMEM/F-12), and fetal bovine serum (FBS) were purchased from HyClone (USA). Endothelial cell medium was obtained from ScienCell (USA). The NBT/BCIP staining kit and detection kit for ALP activity and calcium content were purchased from Beyotime Company (China). The CCK-8 assay was obtained from Dojindo (Japan). MTT, phalloidin, and 4,6-diamidino-2-phenylindole (DAPI) were purchased from AAT Bioquest (USA). Primary antibodies against ALP, BMP-2, RUNX2, OCN, HIF-1 $\alpha$, VEGF, GAPDH, and HRP-conjugated secondary antibodies were obtained from Affinity (China).

Synthesis of $\mathrm{Ca}_{(10-x)} \mathrm{Co}_{x} \mathrm{Li}\left(\mathrm{PO}_{4}\right)_{7}$ powders $(\mathrm{x}=0,0.1,0.25,0.50,1)$

Crystalline $\mathrm{Ca}_{10} \mathrm{Li}\left(\mathrm{PO}_{4}\right)_{7}$ (CLP), in which $\mathrm{Ca}^{2+}$ ions was substituted with different contents of $\mathrm{Co}^{2+}$ ions, was synthesized by a solid-phase sintering method with $\mathrm{Ca}\left(\mathrm{NO}_{3}\right)_{2} \cdot 4 \mathrm{H}_{2} \mathrm{O},\left(\mathrm{NH}_{4}\right)_{2} \mathrm{HPO}_{4}, \mathrm{Li}_{3} \mathrm{PO}_{4}$, and $\mathrm{CoCl}_{2}$ as precursors. The corresponding chemical formulation was $\mathrm{Ca}_{(10-\mathrm{x})} \mathrm{Co}_{\mathrm{x}} \mathrm{Li}\left(\mathrm{PO}_{4}\right)_{7}$, in which $\mathrm{x}=0,0.1,0.25,0.5$, and $1 \mathrm{~mol} \%$. Uniform synthesized powders were accordingly named Co1-CLP, Co2.5-CLP, Co5-CLP, and Co10-CLP. The CLP with no substitution of $\mathrm{Co}^{2+}$ ions for $\mathrm{Ca}^{2+}$ ions, named $\mathrm{Co0}$ CLP, served as the control group and underwent a similar synthetic route. The details of synthetic route are described in $\mathrm{S} 1$.

\section{D printing processes of $\mathrm{Ca} a_{(10-x)} C o_{x} \mathrm{Li}\left(\mathrm{PO}_{4}\right)_{7}$ scaffolds $(x=0,0.1,0.25,0.50,1)$}

Slurry extrusion $3 \mathrm{D}$ printing techniques were used to fabricate porous scaffolds. The slurry extrusion was controlled by the air pressure, which was performed under 0.3 to $0.5 \mathrm{MPa}$. To obtain optimal porous scaffolds, the speed extruding slurry was set as 30 to $40 \mathrm{~mm} / \mathrm{s}$. Porous scaffolds with $10 \mathrm{~mm}$ in diameter and $2 \mathrm{~mm}$ in thickness, abbreviated as Co0, Co1, Co2.5, Co5, and Co10 scaffolds, were obtained after being sintered at $950{ }^{\circ} \mathrm{C}$ for $3 \mathrm{~h}$. The details of the printing processes are described in S2.

\section{Characterization of the Co $(0,1,2.5,5,10)$ scaffolds}

To characterize the Co $(0,1,2.5,5,10)$ scaffolds, the XRD (Miniflex600, Rigaku), Raman spectrophotometer (LabRAM HR, HORIBA), and SEM (SU-8010, HITACHI) were performed to detect crystal phase, surface morphology, and crystal microstructure, respectively.

\section{Compressive strength of the Co $(0,1,2.5,5,10)$ scaffolds}

A testing machine (CMT4304, SANS) was applied to test the compressive strength of the Co $(0,1,2.5,5,10)$ scaffolds $(10 \mathrm{~mm}$ in diameter and $15 \mathrm{~mm}$ in thickness), and a 
constant displacement rate was set as $0.2 \mathrm{~mm} / \mathrm{min}$ according to Chinese Standard GB/T 8489-2006 $(n=4)$.

\section{Mineralization of the $\mathrm{Co}(0,1,2.5,5,10)$ scaffolds in vitro}

SBF was applied to investigate the in vitro mineralization ability of the Co $(0,1,2.5,5,10)$ scaffolds. In detail, the $\mathrm{Co}(0,1,2.5,5,10)$ scaffolds were immersed in $\mathrm{SBF}$ at $37{ }^{\circ} \mathrm{C}$ for 30 days at a ratio of $100 \mathrm{ml} / \mathrm{g}$. The SBF solution was replaced each 3 days. After 30 days of immersion, the scaffolds were removed from the SBF solution, rinsed with distilled water 3 times and dried at $120{ }^{\circ} \mathrm{C}$ for $12 \mathrm{~h}$. SEM (G2, Phenom) and XRD were applied to detect the hydroxyapatite phase on the scaffolds surface.

\section{Degradation properties of the Co $(0,1,2.5,5,10)$ scaffolds in vitro}

To identify the degradation properties, the Co $(0,1,2.5,5,10)$ scaffolds $(n=4)$ were immersed in Tris $-\mathrm{HCl}$ solution at $37^{\circ} \mathrm{C}$ at a ratio of $200 \mathrm{ml} / \mathrm{g}$. After the predetermined time (1, 2, 3, and 4 weeks), a $\mathrm{pH}$ meter was used to test the $\mathrm{pH}$ values of the Tris-HCL solution. Then, the scaffolds were removed from the solution and dried at $120{ }^{\circ} \mathrm{C}$ for $12 \mathrm{~h}$, and their final weights were precisely measured. Weight loss was represented as a percentage of the primary weight.

\section{Cytotoxicity assessment of the Co $(0,1,2.5,5,10)$ scaffolds}

The cytotoxicity of the Co $(0,1,2.5,5,10)$ scaffolds was determined using the MTT assay in mouse fibroblast cells (L-929 cells) according to ISO 10,993-5 2016. To prepare extracts, the Co $(0,1,2.5,5,10)$ scaffolds were soaked in MEM medium at a ratio of $200 \mathrm{mg} / \mathrm{ml}$ at $37^{\circ} \mathrm{C}$ for $24 \mathrm{~h}$. After incubating, the minimum eagle's medium (MEM) was centrifuged, and the resulting supernatant was filtered through a $0.22 \mu \mathrm{m}$ strainer for sterilization.

The optical density (O.D.) of each well was determined using a microplate reader (Multiskan Ascent, Thermo Fisher Scientific) at $570 \mathrm{~nm}$. To calculate the decrease in viability compared with the blank group, the following formula was used: Via. $\%=100 \times O D_{570 \mathrm{e}} / O D_{570 \mathrm{~b}}$ (where $O D_{570 \mathrm{e}}$ is the average value of the measured O.D. of the $100 \%$ extracts, and $O D_{570 \mathrm{~b}}$ is the average value of the measured O.D. of the blanks, $n=6$ ). If viability was decreased to lower than $70 \%$ of the blank, the sample was regarded as showing cytotoxic potential.

\section{Cell culture}

rBMSCs were isolated and cultured according to the approaches described in the previous work [44]. In short, the bone marrow collected from the femurs and tibias of the rats under aseptic conditions was suspended and placed into tissue culture flasks containing Dulbecco's modified eagle's medium/ham's F-12 (DMEM/F-12) supplemented with $10 \%(\mathrm{v} / \mathrm{v})$ FBS and $1 \%(\mathrm{v} / \mathrm{v})$ penicillin/streptomycin solution, and incubated in a $5 \%$ $\mathrm{CO}_{2}$ incubator at $37^{\circ} \mathrm{C}$. The culture medium was replaced every 3 days until the primary rBMSCs were $80 \%$ confluent. The floating hematopoietic cells were removed by multiple medium changes. Subculture was performed through trypsinization when rBMSCs reached $80 \%$ confluence. Only early passage (p3-6) cells were selected for subsequent cellular experiments. 
HUVECs were obtained from iCell Bioscience (China) and cultured in endothelial cell medium (ECM) containing 10\% (v/v) FBS and 1\% (v/v) penicillin/streptomycin solution.

\section{Biocompatibility of the Co $(0,1,2.5,5)$ scaffolds}

\section{The proliferation of $r B M S C s$ cultured on the Co $(0,1,2.5,5)$ scaffolds}

The proliferation of rBMSCs seeded on the Co $(0,1,2.5,5)$ scaffolds was evaluated using the CCK- 8 assay according to the manufacturer's protocol. Briefly, the Co $(0,1,2.5,5)$ scaffolds ( $8 \mathrm{~mm}$ in diameter and $2 \mathrm{~mm}$ in thickness) were first transferred into 48-well plates. Then, rBMSCs were collected and resuspended at $5 \times 10^{4}$ cells $/ \mathrm{ml}$. One hundred microliters of cell suspension was pipetted into each scaffold of 48-well plates and cultured for $1,3,5$, or 7 days. At each preset timepoint, $20 \mu$ of CCK- 8 solution was added to each well and incubated for $2 \mathrm{~h}$ at $37^{\circ} \mathrm{C}$. After incubation, a microplate reader (Thermo Fisher Scientific, USA) was applied to measure the O.D. at $450 \mathrm{~nm}$ in a new 96-well plates. Five data in each group were selected to calculate the average and the standard deviation.

\section{Morphology of rBMSCs cultured on the Co $(0,1,2.5,5)$ scaffolds}

The adhesion and morphology of rBMSCs on the Co $(0,1,2.5,5)$ scaffolds were captured by SEM and CLSM. After coculturing for 3 days, the scaffolds were rinsed in PBS and then fixed in $2.5 \%$ glutaraldehyde solution for $30 \mathrm{~min}$. Then, the cellular scaffolds were dehydrated in an ethanol series and finally dried in a vacuum freeze drier for $3 \mathrm{~h}$. The dehydrated cellular scaffolds were covered with gold, and the morphological characteristics of rBMSCs on the Co $(0,1,2.5,5)$ scaffolds were observed by SEM (FEI Q25, USA).

After 3 and 6 days of culture, the cellular scaffolds were also set in $2.5 \%$ glutaraldehyde solution to fix for $30 \mathrm{~min}$ and then infiltrated with a $0.2 \%$ Triton $\mathrm{X}-100$ solution for $5 \mathrm{~min}$ after rinsing twice with PBS. The samples were then incubated with fluorescein isothiocyanate phalloidin for $1 \mathrm{~h}$ and DAPI for $15 \mathrm{~min}$. The cells were visualized by CLSM (Leica TCS SP8, Germany).

\section{Tubule formation in vitro}

To observe the effect of the Co $(0,1,2.5,5)$ scaffolds on the angiogenic process of HUVECs, we conducted an in vitro tubule formation experiment by coculturing HUVECs with Co $(0,1,2.5,5)$ extracts. First, the Co $(0,1,2.5,5)$ extracts were prepared by soaking the Co $(0,1,2.5,5)$ scaffolds in ECM at a ratio of $200 \mathrm{mg} / \mathrm{ml}$ at $37{ }^{\circ} \mathrm{C}$ for $24 \mathrm{~h}$. Then, the ECM was centrifuged, and the resulting supernatant was filtered through a $0.22 \mu \mathrm{m}$ strainer for sterilization. Briefly, a Matrigel matrix was coated into a 96-well plate for complete gelation for $1 \mathrm{~h}$ at $37^{\circ} \mathrm{C}$. HUVECs were seeded on the Matrigel matrix with Co $(0,1,2.5,5)$ scaffold extracts for 4,6 , and $16 \mathrm{~h}$ of culture. The fields of view per well were imaged by an optical microscope (Leica DMi8, Germany). Five images were analyzed for each group using ImageJ software to quantify total branching length and branches number, which are representative of the angiogenic process in HUVECs.

\section{The effect of the Co $(0,1,2.5,5)$ scaffolds on HIF-1 $a$ and VEGF expression in rBMSCs}

To prepare extracts for rBMSCs culture, the Co $(0,1,2.5,5)$ scaffolds were soaked in DMEM/F-12 medium at a ratio of $200 \mathrm{mg} / \mathrm{ml}$ at $37{ }^{\circ} \mathrm{C}$ for $24 \mathrm{~h}$. After incubation, the 
DMEM/F-12 medium was centrifuged, and the resulting supernatant was filtered through a $0.22 \mu \mathrm{m}$ strainer for sterilization.

A western blot assay was performed to detect HIF-1 $\alpha$ and VEGF expression levels. First, RIPA buffer was added to lyse cells to extract protein after rBMSCs were cultured in Co $(0,1,2.5,5)$ extracts for 7 days, and the protein concentration was quantitated using a BCA Protein Assay Kit. Subsequently, whole-cell lysates were obtained for western blot analysis. Briefly, the same quantity of lysate from the Co $(0,1,2.5,5)$ groups was electrophoresed on SDS-PAGE gels and subsequently transferred onto PVDF membranes. Then, the membranes were blocked in 5\% non-fat milk for $2 \mathrm{~h}$ and incubated with primary antibodies against HIF-1 $\alpha$ (1:1000), VEGF (1:1000), and GAPDH (1:2000) for $24 \mathrm{~h}$ at $4{ }^{\circ} \mathrm{C}$. After rinsing with TBST, the membranes were incubated with HRPconjugated secondary antibodies (1:3000) for $2 \mathrm{~h}$. The band signals of proteins were visualized using enhanced chemiluminescence. The quantitation of band intensity was performed using ImageJ software.

The effect of the Co $(0,1,2.5,5)$ scaffolds on the osteogenic differentiation of rBMSCs alkaline phosphatase (ALP) staining and activity

The preparation of Co $(0,1,2.5,5)$ extracts was the same as that described in Sect. 2.12. Briefly, rBMSCs were seeded in 12-well plates at a density of $1 \times 10^{5}$ cells/well. After rBMSCs reached $80 \%$ confluence, the original medium was replaced by various scaffold extracts. After 7 and 14 days of stimulation, ALP staining and activity assays were performed using an NBT/BCIP staining kit and a detection kit for ALP activity according to a previous protocol. rBMSCs cultured in osteogenic medium were chosen as the positive control group. Five samples in each group were used in these experiments.

\section{Alizarin red staining and calcium content}

After 21 days of stimulation, 4\% paraformaldehyde was used to fix rBMSCs for $30 \mathrm{~min}$ at room temperature. After gentle washing with deionized water, alizarin red solution was added and incubated with rBMSCs for $30 \mathrm{~min}$ to visualize the calcium nodules. The calcium nodules were stained as red spots and imaged by light microscopy (Leica DMi8, Germany). To quantify the mineralization properties of different scaffolds, a detection kit for calcium content was utilized. A standard curve was constructed according to the manufacturer's protocol, and from the equation of the trendline, the calcium content of each sample was calculated with the trendline equation. rBMSCs cultured in osteogenic medium were chosen as the positive control group. Five samples in each group were used in these experiments.

\section{The effect of the Co $(0,1,2.5,5)$ scaffolds on osteogenic protein expression in rBMSCs}

Western blot assay was performed to investigate the efficacy of Co $(0,1,2.5,5)$ extracts on osteogenic protein expression. In brief, rBMSCs were incubated with Co $(0,1,2.5,5)$ extracts for 14 days and lysed in RIPA buffer to extract protein. Then, the protein concentration was determined using a BCA Protein Assay Kit. Subsequently, whole-cell lysates were obtained for western blot analysis. Briefly, the same quantity of proteins from Co $(0,1,2.5,5)$ groups was resolved on SDS-PAGE gels and subsequently transferred to PVDF membranes. Then, the membranes were blocked with $5 \%$ non-fat milk 
for $2 \mathrm{~h}$ and incubated with primary antibodies against ALP (1:1000), BMP-2 (1:1000), RUNX2 (1:1000), OCN (1:1000), and GAPDH (1:2000) for $24 \mathrm{~h}$ at $4{ }^{\circ} \mathrm{C}$. The membranes were then incubated with HRP-conjugated secondary antibodies (1:3000) for $2 \mathrm{~h}$ at room temperature after washing with TBST. The band signals of proteins were detected by enhanced chemiluminescence. The quantification of band intensity was analyzed by ImageJ software.

\section{In vivo bone defect repair}

The experimental protocols for all animals in this study were carried out according to the guidelines approved by the Ethics Committee at the First Affiliated Hospital of Sun Yat-sen University. Co0 and Co2.5 scaffolds with $5 \mathrm{~mm}$ in diameter and $2 \mathrm{~mm}$ in thickness were prepared and sterilized. Eighteen male Sprague-Dawley (SD) rats (Southern Medical University Experiment Animal Center, Guangzhou, China) aged 8 weeks were used and divided into the following three groups $(n=6)$ : the blank group; the $\mathrm{Co} 0$ group; and the Co2.5 group.

\section{Animal surgery and scaffold implantation}

Briefly, $5 \%$ chloral hydrate was managed via intraperitoneal injection for animal anesthesia. A critical bone defect with $5 \mathrm{~mm}$ in diameter in the parietal bone was created in SD rats by an electric trephine drill without injuring the underlying sagittal sinus and dura matter. The $\mathrm{Co} 0$ and $\mathrm{Co} 2.5$ scaffolds filled in the defect, while the blank group received no scaffolds. Then, absorbable sutures were used to stitch the tissue and skin layer by layer. After implantation, the animals were monitored daily by visual observation for signs of inflammation, food intake, activity, and any adverse reaction during the experimental time.

At 8 weeks after the operation, 18 rats were immolated by an overdose of chloral hydrate injected intraperitoneally, and the craniums including the bone defect site with surrounding bone tissue were collected for following analysis. The harvested rat braincase specimens were fixed $10 \%$ neutral-buffered formalin for $24 \mathrm{~h}$ at room temperature and subjected to micro-CT scanning and histological analysis.

\section{Micro-CT analysis}

A micro-CT system (SkyScan 1276, Micro-CT, Bruker, Germany) was applied to scan each braincase, and NRecon software (Bruker, Germany) was used to reconstruct craniums. Then, CTvox software was utilized to perform 3D analyses. A cylindrical region of interest (ROI) with $5 \mathrm{~mm}$ in diameter was outlined at the center of the single defect, completely enclosing the new bone in the defect site. The bone volume $\left(\mathrm{BV} ; \mathrm{mm}^{3}\right)$ and tissue volume (TV; $\mathrm{mm}^{3}$ ) were calculated by assigning a threshold for the total bone content through CTAn analysis software (Skyscan). Then, the bone volume fraction (BV/ TV) was used as a parameter to evaluate the bone formation ability $(n=6)$.

\section{Histological analysis}

Following micro-CT scanning, the harvested craniums were immersed in 10\% EDTA solutions for decalcification until the bone tissue could be readily pierced by needles. After being dehydrated in ascending concentrations of alcohols, the samples 
were embedded in paraffin wax. Four-micron-thick slices were generated by using a microtome. H\&E staining and Masson trichrome staining were performed on selected slices from each specimen to assess bone regeneration. Images of the stained sections were observed by a bright-field microscope (Leica, DMi8, Germany). The percentage of new bone area of each specimen formed in the defects was measured via Image-Pro Plus 6.0 software $(n=6)$.

\section{Statistical analysis}

Statistical analyses were performed using SPSS 23.0 software (IBM, USA). The data were presented as the mean \pm standard deviation (SD) and were analyzed using one-way ANOVA followed by Tukey's post hoc test. A $p$ value $<0.05$ was used as a criterion for statistical significance.

\section{Abbreviations}

B-TCP: Beta-tricalcium phosphate; Co: Cobalt; rBMSCs: Rat bone marrow mesenchymal stem cells; HUVECs: Human umbilical endothelial cells; PDH: Proline hydroxylase: HIF-1a: hypoxia-inducible factor1-alpha; VEGF: Vascular endothelial growth factor: SE-3DP: slurry extrusion-three dimensions printing; ALP: Alkaline phosphatase; BMP-2: Bone morphogenetic protein-2; OCN: Osteocalcin; RUNX2: Runt-related transcription factor 2.

\section{Supplementary Information}

The online version contains supplementary material available at https://doi.org/10.1186/s12938-021-00907-2.

Additional file 1. S1 Synthesis of $\mathrm{Ca}_{(10-x)} \mathrm{Co}_{x} \mathrm{Li}\left(\mathrm{PO}_{4}\right)_{7}$ powders $(x=0,0.1,0.25,0.50,1)$. S2 3D printing processes of $\mathrm{Ca}_{(10-x)} \mathrm{CO}_{x} \mathrm{Li}\left(\mathrm{PO}_{4}\right)_{7}$ scaffolds $(x=0,0.1,0.25,0.50,1)$.

\section{Acknowledgements}

Not applicable.

\section{Authors' contributions}

J: conceptualization, methodology, validation, formal analysis, data curation, writing -original draft preparation, writing —review \& editing, and visualization. CZ: conceptualization, methodology, validation, formal analysis, data curation, writing —original draft preparation, writing — review \& editing, and visualization. CL: writing —original draft preparation, and writing —review \& editing. ZW: resources. ZL: software. BL: investigation. BT: investigation. LZ: investigation. WL: supervision and project administration. XZ: supervision, project administration, and funding acquisition. All authors read and approved the final manuscript.

\section{Funding}

This research was funded by the National Natural Science Foundation of China (32071341, 31430030), Natural Science Foundation of Guangdong Province (2017A030308004), Natural Science Foundation of Guangzhou City (201804020011), The Science and Technology Project of Guangdong province (2018A050506021), and The Joint Funds for the innovation of Science and Technology, Fujian province (2017Y9009, 2017Y9045).

Availability of data and materials

The datasets used and/or analyzed during the current study are available from the corresponding author on reasonable request.

\section{Declarations}

Ethics approval and consent to participate

The study was approved by the Ethics Committee at the First Affiliated Hospital of Sun Yat-sen University (protocol code 2010[94], date of approval: 15 September 2010).

\section{Consent for publication}

All authors consent for the publication of this manuscript.

Competing interests

The authors declare that they have no competing interests.

\section{Author details}

${ }^{1}$ Department of Orthopaedics, Fujian Medical University Union Hospital, Fuzhou 350001, China. ${ }^{2}$ Key Laboratory of Optoelectronic Materials Chemical and Physics, Fujian Institute of Research on the Structure of Matter, Chinese Academy 
of Sciences, Fuzhou, China. ${ }^{3}$ Guangdong Provincial Key Laboratory of Orthopaedics and Traumatology, Department of Spine Surgery, The First Affiliated Hospital of Sun Yat-Sen University, Guangzhou 510080, China.

Received: 23 April 2021 Accepted: 15 July 2021

Published online: 24 July 2021

\section{References}

1. Mebarki M, Coquelin L, Layrolle P, Battaglia S, Tossou M, Hernigou P, et al. Enhanced human bone marrow mesenchymal stromal cell adhesion on scaffolds promotes cell survival and bone formation. Acta Biomater. 2017:59:94-107.

2. Delloye C, Cornu O, Druez V, Barbier O. Bone allografts: What they can offer and what they cannot. J Bone Joint Surg BrVol. 2007;89(5):574-9.

3. Long B, Dan L, Jian L, Yunyu H, Shu H, Zhi Y. Evaluation of a novel reconstituted bone xenograft using processed bovine cancellous bone in combination with purified bovine bone morphogenetic protein. Xenotransplantation. 2012;19(2):122-32.

4. Kargozar S, Lotfibakhshaiesh N, Ai J, Mozafari M, Brouki Milan P, Hamzehlou S, et al. Strontium- and cobalt-substituted bioactive glasses seeded with human umbilical cord perivascular cells to promote bone regeneration via enhanced osteogenic and angiogenic activities. Acta Biomater. 2017;58:502-14.

5. Tanaka T, Komaki H, Chazono M, Kitasato S, Kakuta A, Akiyama S, et al. Basic research and clinical application of betatricalcium phosphate ( $\beta$-TCP). Morphologie : bulletin de l'Association des anatomistes. 2017;101(334):164-72.

6. Tahriri M, Bader R, Yao W, Dehghani S, Khoshroo K, Rasoulianboroujeni M, et al. Bioactive glasses and calcium phosphates. Biomater Oral Dent Tissue Eng. 2018;1:7-24.

7. Takahashi Y, Yamamoto M, Tabata Y. Osteogenic differentiation of mesenchymal stem cells in biodegradable sponges composed of gelatin and beta-tricalcium phosphate. Biomaterials. 2005;26(17):3587-96.

8. Chen Z, Wu C, Gu W, Klein T, Crawford R, Xiao Y. Osteogenic differentiation of bone marrow MSCs by $\beta$-tricalcium phosphate stimulating macrophages via BMP2 signalling pathway. Biomaterials. 2014;35(5):1507-18.

9. Chen Y, Wang J, Zhu XD, Tang ZR, Yang X, Tan YF, et al. Enhanced effect of $\beta$-tricalcium phosphate phase on neovascularization of porous calcium phosphate ceramics: in vitro and in vivo evidence. Acta Biomater. 2015;11:435-48.

10. Bose S, Fielding G, Tarafder S, Bandyopadhyay A. Understanding of dopant-induced osteogenesis and angiogenesis in calcium phosphate ceramics. Trends Biotechnol. 2013;31(10):594-605

11. Saran U, Gemini Piperni S, Chatterjee S. Role of angiogenesis in bone repair. Arch Biochem Biophys. 2014:561:109-17.

12. Kargozar S, Baino F, Hamzehlou S, Hill RG, Mozafari M. Bioactive glasses: sprouting angiogenesis in tissue engineering. Trends Biotechnol. 2018;36(4):430-44.

13. Grasselli F, Basini G, Bussolati S, Bianco F. Cobalt chloride, a hypoxia-mimicking agent, modulates redox status and functional parameters of cultured swine granulosa cells. Reprod Fertil Dev. 2005;17(7):715-20.

14. Duan X, Bradbury SR, Olsen BR, Berendsen AD. VEGF stimulates intramembranous bone formation during craniofacial skeletal development. Matrix Biol. 2016;52-54:127-40.

15. Tahmasebi Birgani Z, Fennema E, Gijbels MJ, de Boer J, van Blitterswijk CA, Habibovic P. Stimulatory effect of cobalt ions incorporated into calcium phosphate coatings on neovascularization in an in vivo intramuscular model in goats. Acta Biomater. 2016:36:267-76.

16. Yilmaz B, Alshemary AZ, Evis Z. Co-doped hydroxyapatites as potential materials for biomedical applications(Review). Microchem J. 2019;144:443-53.

17. Wu C, Zhou Y, Fan W, Han P, Chang J, Yuen J, et al. Hypoxia-mimicking mesoporous bioactive glass scaffolds with controllable cobalt ion release for bone tissue engineering. Biomaterials. 2012;33(7):2076-85.

18. Deng Z, Lin B, Jiang Z, Huang W, Li J, Zeng X, et al. Hypoxia-mimicking cobalt-doped borosilicate bioactive glass scaffolds with enhanced angiogenic and osteogenic capacity for bone regeneration. Int J Biol Sci. 2019;15(6):1113-24

19. Zhao CQ, Xu XC, Lu YJ, Wu SQ, Xu ZY, Huang TT, et al. Doping lithium element to enhance compressive strength of $\beta$-TCP scaffolds manufactured by 3D printing for bone tissue engineering. J Alloy Compd. 2020;814:152327-38.

20. Nagarajan N, Dupret-Bories A, Karabulut E, Zorlutuna P, Vrana NE. Enabling personalized implant and controllable biosystem development through 3D printing. Biotechnol Adv. 2018;36(2):521-33.

21. Ma T, Jee JB, Silva SA, Blomgren CB, Pontinha VM, Dixon DL, et al. Additive manufacturing of pharmaceuticals for precision medicine applications: A review of the promises and perils in implementation(Review). Addit Manuf. 2018:23:319-28

22. Kannan RY, Salacinski HJ, Sales K, Butler P, Seifalian AM. The roles of tissue engineering and vascularisation in the development of micro-vascular networks: a review. Biomaterials. 2005;26(14):1857-75.

23. Sun $X$, Kang $Y$, Bao J, Zhang $Y$, Yang $Y$, Zhou X. Modeling vascularized bone regeneration within a porous biodegradable CaP scaffold loaded with growth factors. Biomaterials. 2013;34(21):4971-81.

24. Bbyset $Y$, Ammar Z, Zafer A. Co-doped hydroxyapatites as potential materials for biomedical applications (Review). J Microchem J. 2019;1:443-53.

25. Laurencin D, Almora-Barrios N, de Leeuw NH, Gervais C, Bonhomme C, Mauri F, et al. Magnesium incorporation into hydroxyapatite. Biomaterials. 2011;32(7):1826-37.

26. Tank KP, Chudasama KS, Thaker VS, Joshi MJ. Cobalt-doped nanohydroxyapatite: Synthesis, characterization, antimicrobial and hemolytic studies. J Nanopart Res. 2013;15(55):1644-55.

27. Cheng G, Deng C, Wu C, Yin H, Ruan Y, Sun Y, Xie Q, Wu X. Effects of Mn-doping on the structural evolution of $\beta$-Tricalcium Phosphate by Rietveld refinement and Raman spectroscopy. Mater Lett. 2019;235:236-8. 
28. Oonishi H, Hench LL, Wilson J, Sugihara F, Tsuji E, Kushitani S, et al. Comparative bone growth behavior in granules of bioceramic materials of various sizes. J Biomed Mater Res. 1999;44(1):31-43.

29. de Siqueira L, de Paula CG, Gouveia RF, Motisuke M, de Sousa TE. Evaluation of the sintering temperature on the mechanical behavior of $\beta$-tricalcium phosphate/calcium silicate scaffolds obtained by gelcasting method. J Mech Behav Biomed Mater. 2019;90:635-43.

30. Kawashita M, Shineha R, Kim HM, Kokubo T, Inoue Y, Araki N, et al. Preparation of ceramic microspheres for in situ radiotherapy of deep-seated cancer. Biomaterials. 2003;24(17):2955-63.

31. Zhang L, Yang G, Johnson BN, Jia X. Three-dimensional (3D) printed scaffold and material selection for bone repair. Acta Biomater. 2019;84:16-33.

32. Roseti L, Parisi V, Petretta M, Cavallo C, Desando G, Bartolotti I, et al. Scaffolds for bone tissue engineering: state of the art and new perspectives. Mater Sci Eng, C Mater Biol Appl. 2017;78:1246-62.

33. Daou S, El Chemaly A, Christofilopoulos P, Bernard L, Hoffmeyer P, Demaurex N. The potential role of cobalt ions released from metal prosthesis on the inhibition of $\mathrm{Hv} 1$ proton channels and the decrease in Staphyloccocus epidermidis killing by human neutrophils. Biomaterials. 2011;32(7):1769-77.

34. Clarke B. Normal bone anatomy and physiology. Clin J Am Soc Nephrol. 2008;3(Suppl 3):S131-9.

35. Buttyan R, Chichester P, Stisser B, Matsumoto S, Ghafar MA, Levin RM. Acute intravesical infusion of a cobalt solution stimulates a hypoxia response, growth and angiogenesis in the rat bladder. J Urol. 2003;169(6):2402-6.

36. Hewitson KS, McNeill LA, Riordan MV, Tian YM, Bullock AN, Welford RW, et al. Hypoxia-inducible factor (HIF) asparagine hydroxylase is identical to factor inhibiting $\mathrm{HIF}(\mathrm{FIH})$ and is related to the cupin structural family. J Biol Chem. 2002;277(29):26351-5.

37. Zou D, Zhang Z, He J, Zhang K, Ye D, Han W, et al. Blood vessel formation in the tissue-engineered bone with the constitutively active form of HIF-1 a mediated BMSCs. Biomaterials. 2012:33(7):2097-108.

38. Soker S, Machado M, Atala A. Systems for therapeutic angiogenesis in tissue engineering. World J Urol. 2000;18(1):10-8.

39. Komori T. Regulation of proliferation, differentiation and functions of osteoblasts by Runx2. Int J Mol Sci. 2019;20(7):1694-705.

40. Hu X, Yu SP, Fraser JL, Lu Z, Ogle ME, Wang JA, et al. Transplantation of hypoxia-preconditioned mesenchymal stem cells improves infarcted heart function via enhanced survival of implanted cells and angiogenesis. J Thorac Cardiovasc Surg. 2008;135(4):799-808.

41. Kulanthaivel S, Roy B, Agarwal T, Giri S, Pramanik K, Pal K, et al. Cobalt doped proangiogenic hydroxyapatite for bone tissue engineering application. Mater Sci Eng, C Mater Biol Appl. 2016;58:648-58.

42. Fan W, Crawford R, Xiao Y. Enhancing in vivo vascularized bone formation by cobalt chloride-treated bone marrow stromal cells in a tissue engineered periosteum model. Biomaterials. 2010:31(13):3580-9.

43. Oladipupo S, Hu S, Kovalski J, Yao J, Santeford A, Sohn RE, et al. VEGF is essential for hypoxia-inducible factor-mediated neovascularization but dispensable for endothelial sprouting. Proc Natl Acad Sci USA. 2011:108(32):13264-9.

44. Soleimani M, Nadri S. A protocol for isolation and culture of mesenchymal stem cells from mouse bone marrow. Nat Protoc. 2009;4(1):102-6.

\section{Publisher's Note}

Springer Nature remains neutral with regard to jurisdictional claims in published maps and institutional affiliations.

- fast, convenient online submission

- thorough peer review by experienced researchers in your field

- rapid publication on acceptance

- support for research data, including large and complex data types

- gold Open Access which fosters wider collaboration and increased citations

- maximum visibility for your research: over $100 \mathrm{M}$ website views per year

At BMC, research is always in progress.

Learn more biomedcentral.com/submissions 